UDC 547.56.56.563:264

\title{
SYNTHESIS OF NOVEL MANNICH BASES ON THE BASE OF 1-PHENOXY-3-PROPYLTHIOPROPANE-2-OL AND SECONDARY AMINES
}

\author{
I.A.Jafarov ${ }^{1}$, E.H.Mammadbayli ${ }^{2}$, K.A.Kochetkov ${ }^{3}$, A.D.Astanova ${ }^{1}$, G.M.Talybov ${ }^{2}$ \\ ${ }^{1}$ Azerbaijan Pedagogical University \\ ${ }^{2}$ Institute of Petrochemical Processes, NAS of Azerbaijan \\ ${ }^{3}$ Institute of Elementorganic Compounds Russian Academy of Sciences, Moscow
(n)
}

eldar_mamedbeyli@mail.ru

Received 11.12.2018

\begin{abstract}
The condensation of 1-phenoxy-3-propylthiopropan-2-ol with formaldehyde and secondary aliphatic, as well as heterocyclic amines, has led to the formation of new aminomethoxy derivatives of 1-phenoxy-3propylthiopropane with $69-77 \%$ yields. The physico-chemical parameters of the synthesized compounds were determined, their structure was confirmed by the methods of elemental analysis, IR spectroscopy, ${ }^{1} \mathrm{H}$ and ${ }^{13} \mathrm{C}$ NMR. The compounds obtained were tested as antiseptic substances against bacteria and fungi. It is established that they are more effective than the currently used medical drugs.
\end{abstract}

Keywords: aminomethoxy derivatives, 1-phenoxy-3-propylthiopropan-2-ol formaldehyde, secondary amines, diethylamine, dibutylamine, piperidine, morfoline.

https://doi.org/10.32737/0005-2531-2019-2-29-34

\section{Introduction}

Organic compounds containing various functional groups and heteroatoms, such as sulfur and nitrogen, play an enormous role both in the development of synthetic organic chemistry and in applied research. They are widely used as effective biologically active substances and pharmaceuticals, as well as additives to improve the quality of oils and fuels [1-3]. The task of purposeful synthesis of such compounds used in various chemical fields is highly relevant. In this regard, the synthesis of new generations of such compounds based on available raw materials and improved methods continues to attract the attention of researchers $[4,5]$. Since biologically active compounds, which combine several pharmacophoric fragments, have more effective actions, researchers try to expand the range of such compounds and study their physiologically active properties $[6,7]$. One of the convenient and promising methods for the synthesis of new sulfurcontaining aminomethoxy derivatives is the threecomponent Mannich reaction, since the use of a wide range of suitable starting compounds with an active methylene group, as well as various amine and aldehyde components, allows to obtain an extensive range of multifunctional derivatives of this class of compound [8]. The multicomponent Mannich reaction is an ecologically pure and classical method of obtaining biologically active and medical preparations, and is one of the most important and promising reactions of organic chemistry [9]. Aminomethyl derivatives of various classes of organic compounds exhibit antitumor activity [10], affect the cardiovascular system, cause a decrease in blood pressure, are used as drugs for the treatment of Parkinson's disease, are part of antimalarial drugs, exhibit antidepressant properties [11], and their effective acting as antispasmodic, anesthetic and diuretic substances [12].

\section{Discussion of the obtained results}

This paper presents the results of the synthesis and study of the properties of new aminomethoxy derivatives of 1-phenoxy-3-propylthiopropane (IX-XIII). At the first stage, the initial sulfur-containing secondary alcohol, 1-phenoxy-3-propylthiopropan-2-ol (III), was synthesized by reaction of phenol (I) with 1-chloro-3propylthiopropan-2-ol (II). The reaction goes according to the Scheme 1:

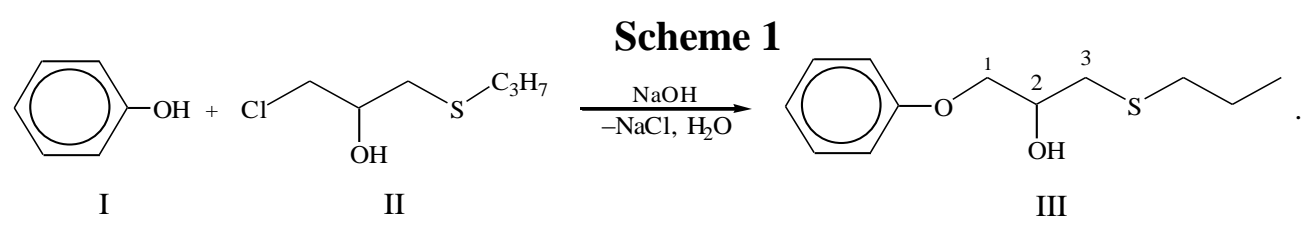


The reaction was carried out at an equimolar ratio of the initial reagents, $75-80^{\circ} \mathrm{C}$, duration $4 \mathrm{~h}$, and in an aqueous solution of $\mathrm{NaOH}(40 \%)$. The yield of the whole product was $70 \%$. New representatives of aminometh- oxy derivatives of 1-phenoxy-3-propylthiopropane IX-XIII were synthesized by the interaction of 1-phenoxy-3-propylthiopropan-2-ol III with formaldehyde and secondary amines (IVVIII) according to the Scheme 2:

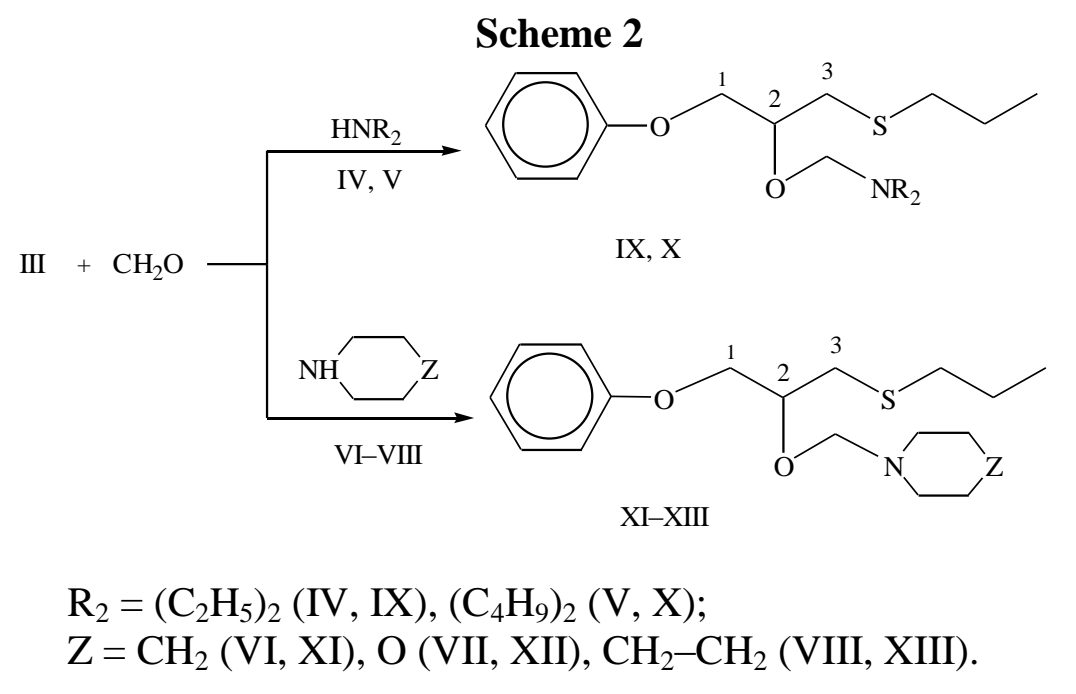

The synthesis of aminomethoxy derivatives IX-XIII by Mannich condensation was carried out at a temperature of $45-50^{\circ} \mathrm{C}$ for $3-4 \mathrm{~h}$, the yield was $69-77 \%$. The physico-chemical parameters of the synthesized compounds were determined. The compounds obtained XI-XIII are liquids with a characteristic odor, insoluble in water, well soluble in organic solvents (ethanol, acetone, benzene, $\mathrm{CCl}_{4}, \mathrm{CHCl}_{3}$ etc.). The composition and structure of the compounds obtained III, IX-XIII were confirmed using elemental analysis data, IR spectroscopy, ${ }^{1} \mathrm{H}$ and ${ }^{13} \mathrm{C}$ NMR, as well as mass-spectrometry. The identity of the original and synthesized compounds, as well as the composition of the reaction mixtures was monitored by GLC.

In the IR spectrum of compound III, a wide absorption band is observed in the region of $3400 \mathrm{~cm}^{-1}$ characteristic of the valent vibration of the hydroxyl group $\left(\mathrm{v}_{\mathrm{OH}}\right)$ of the secondary alcohol [13], which is absent in the corresponding spectra of compounds IX-XIII. For all synthesized compounds, the absorption bands in the range of $737-730 \mathrm{~cm}^{-1}$, characteristic of stretching vibrations of the $\mathrm{C}-\mathrm{S}$ bond, were detected. In the spectra of these compounds there are intense absorption bands of deformation vibrations $\delta_{\mathrm{C}-\mathrm{H}}$ in the range of $700-650 \mathrm{~cm}^{-1}$.
Along with this, bands in the region of 2920 2890, 2880-2840 $\mathrm{cm}^{-1}$, characteristic for the $\mathrm{CH}_{3}$ and $\mathrm{CH}_{2}$ groups, respectively, were found. The $v_{\mathrm{C}-\mathrm{N}}$ stretching vibrations for compounds IX-XIII manifest themselves in the region of $1140-1130 \mathrm{~cm}^{-1}$. The stretching vibrations of the $\mathrm{C}-\mathrm{O}$ bond $\left(v_{\mathrm{C}-\mathrm{O}}\right)$ manifest themselves in the region of $1100-1050 \mathrm{~cm}^{-1}$ in the form of a band of average intensity.

The ${ }^{1} \mathrm{H}$ and ${ }^{13} \mathrm{C}$ NMR spectra of the synthesized compounds III, IX-XIII also confirm the indicated structure (Scheme 1,2). In the mass spectra of the synthesized compounds, the signals of the corresponding molecular ions, as well as the products of their fragmentation, are noted.

The compounds were tested as antimicrobial additives for lubricating oil M-11. The test results showed that compounds IX-XIII have bactericidal and fungicidal properties and effectively act to suppress the growth of microorganisms in M-11 oil at a concentration of 0.5 and $1.0 \%$, while compounds X and XII have a higher efficiency, than other compounds, and higher than that of industrial additives Napentachlorophenolate, taken as ethanol. The remaining compounds show close to the standard results. 
Compounds IX-XIII were tested for antimicrobial activity. The study of the antimicrobial properties of the compounds was carried out in comparison with the preparations used in practice: ethanol, carbolic acid (phenol), chloramines, rivanol, nitrofungin. Antimicrobial activity of substances was studied by the method of serial dilutions. Gram-negative (intestinal and Pseudomonas aeruginosa), gram-positive (Staphylococcus aureus), sporiferous (anthracoid) bacteria and yeastlike fungi (Candida genus) were taken as testcultures. The results obtained in the study of antimicrobial activity showed that the test compounds: 1-phenoxy-3-propylthio-2-(N,N-diethylaminomethoxy)propane (IX), 1-phenoxy-3-propylthio-2-(N,N-dibutylaminomethoxy)propane (X), 1-phenoxy-3-propylthio-2-piperidinemethoxypropane (XI), 1-phenoxy-3-propylthio-2-morpholinomethoxypropane (XII) exhibit a more pronounced antimicrobial activity than the practical use of alcohol, carbolic acid, rivanol, nitrofungin and furacilin. These compounds can be recommended as antimicrobial agents.

\section{Experimental part}

The IR spectra of the synthesized compounds were recorded on a "Spectrum BX" and "Bruker" apparatus of the "ALPHA IR FURYE" company (Germany) in the region of 4000-400 $\mathrm{cm}^{-1}$. The spectra ${ }^{1} \mathrm{H}$ and ${ }^{13} \mathrm{C}$ NMR were recorded on a "Bruker" AM-300 spectrometer, at a frequency of $300 \mathrm{MHz}$, solvent $\mathrm{C}_{6} \mathrm{D}_{6}$, internal standard HMDS.

The purity of the reaction products was determined by the boiling point, elemental analysis data and gas-liquid chromatography.

Elemental analysis was carried out on a "CARLOERBA" instrument, model EA 1108. GLC analysis was performed on an LXM-MD chromatograph, a steel column $(300 \times 3 \mathrm{~mm})$ with 5\% PEGS (polyethyleneglycolsuccinate) on dinochrome II, carrier gas - helium (40 $\mathrm{cm}^{3} / \mathrm{min}$ ), katharometer detector, column temperature $150^{\circ} \mathrm{C}$, evaporator $-230^{\circ} \mathrm{C}$, refractive index was determined on an "ABBEMAT" 350/500 refractometer, density - on a device DMA 4500M.

Compound III, formaldehyde and secondary amines: diethylamine (IV), dibutylamine
(V), piperidine (VI), morpholine (VII), hexamethylenimine (VIII) were used for the synthesis of aminomethoxy derivatives of 1-phenoxy3-propylthiopropane IX-XIII. All amines (IVVIII) were used as reactives. Before use, the amines were distilled and determined their physicochemical constants, which coincide with the literature data.

Ammonia water, which is a medicine, was used in the form of a $10 \%$ solution. Benzene was used as a solvent, which was purified and dried by a known method [14].

Paraformaldehyde, a formaldehyde polymerization product consisting of 8-100 monomers, is depolymerized to formaldehyde when heated and used in the form of a reactive powder.

The starting 1-chloro-3-propanylthiopropan-2-ol (II) was obtained according to the method [14].

The effect of compounds IX-XIII as antimicrobial additives to $\mathrm{M}-11$ oil was studied using their solutions in oil with a concentration of $0.5-1.0 \%$. Antimicrobial properties were determined in a thermogram according to GOST 9025-74 and 9052-75, as well as by the Hole method at a temperature of $28-30^{\circ} \mathrm{C}$ for $2-3$ days. Fungal (Aspergillus niger, Candida tropicalis) and bacterial (Mycobacterium lacticola, Pseudomonas aeruginoza) cultures were used as test organisms.

The antimicrobial activity of compounds IX-XIII was studied by the serial dilution method on several strains of microorganisms. MPA with $\mathrm{pH}$ 7.2-7.4 (for bacteria) and Saburo's medium (for fungi) were used as nutrients. The dilution rates were 1:200, 1:400, 1:800, 1:1600 and 1:3200. As standards for comparison, alcohol, phenol, chloramine, rivanol, and nitrofungin were investigated in the same dilutions. Seeding was carried out after 10, 20, 30, 40 and 60 minutes for bacteria and fungi.

1-Phenoxy-3-propylthiopropan-2-ol (III). To a mixture of $23.5 \mathrm{~g}(0.25 \mathrm{~mol})$ of phenol I and $25 \mathrm{~g}$ of a $40 \%$ aqueous solution of $\mathrm{NaOH}$ at $75-80^{\circ} \mathrm{C}$ and vigorous stirring were added drop wise $42.17 \mathrm{~g}(0.25 \mathrm{~mol})$ of 1-chloro-3propanylthiopropane-2-ol (II). Stirring was continued for 3-4 hours. After cooling, benzene was added to the mixture, the organic layer was 
separated and washed with $5 \% \mathrm{NaOH}$ solution, then with water until neutral, dried with $\mathrm{MgSO}_{4}$. After distilling off the solvent, the residue was distilled in vacuum. Yield $-39.6 \mathrm{~g}$ (70\%) of 1-phenoxy-3-propylthiopropan-2-ol (III), b.p. $-149-152^{0} \mathrm{C}(2 \mathrm{~mm} \mathrm{Hg}), n_{D}^{20}-$ 1.5446, $d_{4}^{20}-1.08692$. $M R_{D}$ found 65.66 , calculated 65.52. IR spectrum, $v, \mathrm{~cm}^{-1}: 735(\mathrm{C}-\mathrm{S})$, $3340(\mathrm{OH}), 2850\left(\mathrm{CH}_{2}\right), 2930\left(\mathrm{CH}_{3}\right) . \mathrm{NMR}$ spectrum ${ }^{1} \mathrm{H}, \delta$, ppm: $0.98 \mathrm{t}\left(3 \mathrm{H}, \mathrm{CH}_{3}, J=7.1\right.$ $\mathrm{Hz}), 1.2 \mathrm{t}\left(2 \mathrm{H}, \mathrm{CH}_{2}, J=7.1 \mathrm{~Hz}\right), 2.49$ d.d $(1 \mathrm{H}$, $\left.\mathrm{CH}_{2} \mathrm{~S}, J=14 \mathrm{~Hz}, J=7.7 \mathrm{~Hz}\right), 2.61$ d.d $\left(1 \mathrm{H}, \mathrm{CH}_{2} \mathrm{~S}\right.$, $J=14.0 \mathrm{~Hz}, J=7.7 \mathrm{~Hz}), 2.7 \mathrm{~d}\left(2 \mathrm{H}, \mathrm{CH}_{2} \mathrm{~S}\right), 3.01 \mathrm{~s}$ $(1 \mathrm{H}, \mathrm{OH}), 7.15-7.68 \mathrm{~m}\left(5 \mathrm{H}, \mathrm{C}_{6} \mathrm{~N}_{5}\right)$. NMR spectrum ${ }^{13} \mathrm{C}, \delta$, ppm: $14.4,15.2,17.5,18.2,20.4$, 35.8, 37.4, 38.6, 69.5, 86.4, 88.15. [M] - 226, calculated - 226.34. Found, \%: C 63.47, H 7.95, $\mathrm{S}$ 14.09. $\mathrm{C}_{12} \mathrm{H}_{18} \mathrm{O}_{2} \mathrm{~S}$. Calculated, \%: C 63.68, H 8.02, S 14.17.

Aminomethoxy derivatives of 1-phenoxy-3-propylthiopropane (IX-XIII). General method of synthesis. To a solution of $0.03 \mathrm{~mol}$ of alcohol and $0.03 \mathrm{~mol}$ of formaldehyde (obtained from paraform during the reaction) in 30 $\mathrm{ml}$ of dry benzene was added dropwise at 20$22^{\circ} \mathrm{C}$ and stirring $0.03 \mathrm{~mol}$ of freshly distilled amine (IV-VIII). Stirring was continued at 45$50^{\circ} \mathrm{C}$ for $3-4 \mathrm{~h}$. After the solvent was distilled off, the residue was distilled in vacuum.

1-Phenoxy-3-propylthio-2-(N,N-diethylaminomethoxy)propane (IX) was prepared from $6.78 \mathrm{~g}(0.03 \mathrm{~mol})$ of compound III, $0.9 \mathrm{~g}$ $(0.03 \mathrm{~mol})$ of formaldehyde and $2.19 \mathrm{~g}(0.03$ mol) of diethylamine (IV). Yield $-6.43 \mathrm{~g}(69 \%)$, b.p. $-163-165^{\circ} \mathrm{C}(1 \mathrm{~mm} \mathrm{Hg}), n_{D}^{20}-1.5144$, $d_{4}^{20}-1.0153 . M R_{D}$ found 65.66, calculated 65.52. IR spectrum, $v, \mathrm{~cm}^{-1}: 3070\left(\mathrm{C}-\mathrm{N}_{\text {arom }}\right)$, $2900\left(\mathrm{CH}_{3}\right), 2840\left(\mathrm{CH}_{2}\right), 1600\left(\mathrm{C}=\mathrm{C}_{\text {arom }}\right), 1200$ $(\mathrm{C}-\mathrm{N}), 1100(\mathrm{C}-\mathrm{O}), 735(\mathrm{C}-\mathrm{S})$. NMR spectrum ${ }^{1} \mathrm{H}, \delta, p p m: 1.01 \mathrm{t}\left(6 \mathrm{H}, 2 \mathrm{CH}_{3}, J=7.4 \mathrm{~Hz}\right), 1.08 \mathrm{t}$ $\left(3 \mathrm{H}, \mathrm{CH}_{3}, J=7.2 \mathrm{~Hz}\right), 1.21 \mathrm{t}\left(2 \mathrm{H}, \mathrm{CH}_{2}, J=7.2\right.$ $\mathrm{Hz}), 1.32-1.41 \mathrm{~m}\left(4 \mathrm{H}, 2 \mathrm{CH}_{2}\right), 3.03 \mathrm{k}(4 \mathrm{H}$, $\left.2 \mathrm{CH}_{2} \mathrm{~N}\right), 3.62 \mathrm{~m}\left(2 \mathrm{H}, \mathrm{OCH}_{2}\right), 4.04-4.11 \mathrm{~m}(1 \mathrm{H}$, $\mathrm{SNO}), 4.59 \mathrm{~s}\left(2 \mathrm{H}, \mathrm{OCH}_{2} \mathrm{~N}\right), 7.15-7.34 \mathrm{~m}(5 \mathrm{H}$, $\left.\mathrm{C}_{6} \mathrm{H}_{5}\right)$. NMR ${ }^{13} \mathrm{C}, \delta$, ppm: 14.4, 15.2, 17.5, 18.2, 20.2, 35.4, 37.4, 38.6, 39.6, 69.5, 86.4, 88.5.
[M] - 311, calculated - 311.48. Found, \%: C 65.45, H 9.31, N 4.45; $\mathrm{S} 10.21 . \mathrm{C}_{17} \mathrm{H}_{29} \mathrm{NO}_{2} \mathrm{~S}$. Calculated, \%: C 65.56, H 9.38, N 4.50, S 10.29.

\section{1-Phenoxy-3-propylthio-2-(N,N-dibutyl-} aminomethoxy)propane $(\mathbf{X})$ was prepared from $6.78 \mathrm{~g}(0.03 \mathrm{~mol})$ of compound III, $0.9 \mathrm{~g}(0.03$ $\mathrm{mol})$ of formaldehyde and $3.87 \mathrm{~g}(0.03 \mathrm{~mol})$ of dibutylamine (V) Yield - $8.47 \mathrm{~g}(77 \%)$, b.p. $198-199^{\circ} \mathrm{C}(1 \mathrm{~mm} \mathrm{Hg}), n_{D}^{20}-1.5140, d_{4}^{20}-$ 1.0788. $M R_{D}$ found 111.21, calculated 111.36. IR spectrum, $v, \mathrm{~cm}^{-1}: 3060\left(\mathrm{C}-\mathrm{N}_{\text {arom }}\right), 2910$ $\left(\mathrm{CH}_{3}\right), 2850\left(\mathrm{CH}_{2}\right), 1585\left(\mathrm{C}=\mathrm{C}_{\text {arom. }}\right), 1200(\mathrm{C}-$ N), $1050(\mathrm{C}-\mathrm{O}), 735(\mathrm{C}-\mathrm{S})$. NMR spectrum ${ }^{1} \mathrm{H}$, $\delta$, ppm: $0.98 \mathrm{t}\left(6 \mathrm{H}, 2 \mathrm{CH}_{3}, J=7.1 \mathrm{~Hz}\right), 1.01 \mathrm{t}(3 \mathrm{H}$, $\left.\mathrm{CH}_{3}, J=7.1 \mathrm{~Hz}\right), 1.21-1.32 \mathrm{~m}\left(10 \mathrm{H}, 5 \mathrm{CH}_{2}\right)$, 1.45-1.51 m $\left(2 \mathrm{H}, \mathrm{CH}_{2}\right), 1.65 \mathrm{~m}\left(2 \mathrm{H}, \mathrm{CH}_{2}\right), 2.49$ d.d $\left(1 \mathrm{H}, \mathrm{CH}_{2} \mathrm{~S}, J=14.0 \mathrm{~Hz}, J=7.7 \mathrm{~Hz}\right) 2.61$ d.d $\left(1 \mathrm{H}, \mathrm{CH}_{2} \mathrm{~S}, J=14.0 \mathrm{~Hz}, J=7.7 \mathrm{~Hz}\right), 3.03 \mathrm{t}(4 \mathrm{H}$, $\left.2 \mathrm{CH}_{2} \mathrm{~N}\right), 3.62 \mathrm{~m}\left(2 \mathrm{H}, \mathrm{CH}_{2} \mathrm{O}\right), 4.04 \mathrm{~m}(1 \mathrm{H}$, $\mathrm{CHO}), 4.59$ c $\left(2 \mathrm{H}, \mathrm{OCH}_{2} \mathrm{~N}\right), 7.15-7.34 \mathrm{~m}(5 \mathrm{H}$, $\mathrm{C}_{6} \mathrm{H}_{5}$ ). NMR spectrum ${ }^{13} \mathrm{C}, \delta$, ppm: $14.4,15.2$, $16.4,18.2,20.2,35.4,38.6,69.9,86.4,88.5$. [M] - 367, calculated - 367.59. Found, \%: C 68.57, $\mathrm{H} 10.25, \mathrm{~N} 3.7, \mathrm{~S} 8.65 . \mathrm{C}_{21} \mathrm{H}_{37} \mathrm{NO}_{2} \mathrm{~S}$. Calculated, $\%$ : C 68.62, H 10.15, N 3.81, S 8.72.

1-Phenoxy-3-propylthio-2-piperidinomethoxypropane (XI) was obtained from $6.78 \mathrm{~g}$ $(0.03 \mathrm{~mol})$ of compound III, $0.9 \mathrm{~g}(0.03 \mathrm{~mol})$ of formaldehyde and $2.55 \mathrm{~g}(0.03 \mathrm{~mol})$ of piperidine (VI). Yield - 6.97 g (72\%), b.p. - 190$192^{0} \mathrm{C}(1 \mathrm{~mm} \mathrm{Hg}), n_{D}^{20}-1.5294, d_{4}^{20}-1.0456$. $M R_{D}$ found 95.46 , calculated 95.37. IR spectrum, $v, \mathrm{~cm}^{-1}$ : $3050\left(\mathrm{C}-\mathrm{N}_{\text {arom. }}\right), 2895\left(\mathrm{CH}_{3}\right)$, $2850\left(\mathrm{CH}_{2}\right), 1585\left(\mathrm{C}=\mathrm{C}_{\text {arom }}\right), 1250(\mathrm{C}-\mathrm{N}), 1050$ (C-O), $650(\mathrm{C}-\mathrm{S})$. NMR spectrum ${ }^{1} \mathrm{H}, \delta$, ppm: $1.01 \mathrm{t}\left(3 \mathrm{H}, \mathrm{CH}_{3}, J=7.3 \mathrm{~Hz}\right), 1.21 \mathrm{~m}\left(2 \mathrm{H}, \mathrm{CH}_{2}\right)$, $1.32 \mathrm{~m}\left(6 \mathrm{H}, 3 \mathrm{CH}_{2 \text { cyc. }}\right), 2.49$ d.d $\left(1 \mathrm{H}, \mathrm{CH}_{2} \mathrm{~S}\right.$, $J=14.0 \mathrm{~Hz}, J=7.7 \mathrm{~Hz}), 2.61$ d.d $\left(1 \mathrm{H}, \mathrm{CH}_{2} \mathrm{~S}\right.$, $J=14.0 \mathrm{~Hz}, J=7.7 \mathrm{~Hz}), 3.01 \mathrm{t}\left(4 \mathrm{H}, 2 \mathrm{CH}_{2} \mathrm{~N}\right), 3.62$ $\mathrm{m}\left(2 \mathrm{H}, \mathrm{OCH}_{2}\right), 4.04 \mathrm{~m}(1 \mathrm{H}, \mathrm{OCH}), 4.51 \mathrm{~s}(2 \mathrm{H}$, $\left.\mathrm{OCH}_{2} \mathrm{~N}\right), 7.15-7.34 \mathrm{~m}\left(5 \mathrm{H}, \mathrm{C}_{6} \mathrm{H}_{5}\right) . \mathrm{NMR}{ }^{13} \mathrm{C}, \delta$, ppm: 14.4, 15.2, 17.5, 20.45, 26.21, 35.4, 36.4, $39.6,69.9,86.4,88.5$. [M] - 323, calculated 323.49. Found, \%: C 66.92, H 8.96, N 4.28, S 9.80. $\mathrm{C}_{18} \mathrm{H}_{29} \mathrm{NO}_{2}$ S. Calculated, \%: C 66.83, H 9.04, N 4.33, S 9.91. 
1-Phenoxy-3-propylthio-2-morpholinomethoxypropane (XII) was obtained from 6.78 $\mathrm{g}(0.03 \mathrm{~mol})$ of compound III, $0.9 \mathrm{~g}(0.03 \mathrm{~mol})$ of formaldehyde, and $2.61 \mathrm{~g}(0.03 \mathrm{~mol})$ of morpholine (VII). Yield - $7.21 \mathrm{~g}(74 \%)$, b.p. - 192$194^{0} \mathrm{C}(1 \mathrm{~mm} \mathrm{Hg}), n_{D}^{20}-1.5312, d_{4}^{20}-1.0889$. $M R_{D}$ found 92.50 , calculated 92.48 . IR spectrum, $v, \mathrm{~cm}^{-1}: 3060$ (C-N arom.), $2900\left(\mathrm{CH}_{3}\right), 2840$ $\left(\mathrm{CH}_{2}\right), 1600,1500\left(\mathrm{C}=\mathrm{C}_{\text {arom}}\right), 1250(\mathrm{C}-\mathrm{N}), 1100$ (C-O), $750(\mathrm{C}-\mathrm{S})$. NMR spectrum ${ }^{1} \mathrm{H}, \delta$, ppm: $1.01 \mathrm{t}\left(3 \mathrm{H}, \mathrm{CH}_{3}, J=7.1 \mathrm{~Hz}\right), 1.21-1.32 \mathrm{~m}(4 \mathrm{H}$, $\left.2 \mathrm{CH}_{2}\right), 1.45 \mathrm{~m}\left(2 \mathrm{H}, \mathrm{CH}_{2}\right), 2.49$ d.d $\left(2 \mathrm{H}, \mathrm{CH}_{2} \mathrm{~S}\right.$, $J=14.0 \mathrm{~Hz}, J=7.7 \mathrm{~Hz}), 2.61$ d.d $\left(2 \mathrm{H}, \mathrm{CH}_{2} \mathrm{~S}\right)$, $3.03 \mathrm{t}\left(4 \mathrm{H}, 2 \mathrm{CH}_{2} \mathrm{~N}\right), 3.75 \mathrm{~m}\left(4 \mathrm{H}, 2 \mathrm{CH}_{2} \mathrm{O}_{\text {cyc. }}\right)$, $3.82 \mathrm{~m}\left(2 \mathrm{H}, \mathrm{OCH}_{2}\right), 4.04 \mathrm{~m}(1 \mathrm{H}, \mathrm{CHO}), 4.51 \mathrm{~s}$ $\left(2 \mathrm{H}, \mathrm{OCH}_{2} \mathrm{~N}\right), 7.13-7.36 \mathrm{~m}\left(5 \mathrm{H}, \mathrm{C}_{6} \mathrm{H}_{5}\right)$. NMR ${ }^{13} \mathrm{C}, \delta$, ppm: 14.4, 15.2, 17.5, 19.2, 20.45, 26.21, $35.4,36.4,39.6,69.6,69.9,86.4,88.15$. [M] 325 , calculated -325.47 . Found, \%: C 62.52, H 8.27, N 4.26, S 9.74. $\mathrm{C}_{17} \mathrm{H}_{27} \mathrm{NO}_{3}$ S. Calculated, $\%$ : C 62.73, H 8.36, N 4.30, S 9.85.

1-Phenoxy-3-propylthio-2-hexamethylenimnomethoxypropane (XIII) was obtained from $6.78 \mathrm{~g}(0.03 \mathrm{~mol})$ of compound III, $0.9 \mathrm{~g}$ $(0.03 \mathrm{~mol})$ of formaldehyde, and $2.97 \mathrm{~g}(0.03$ mol) of hexamethyleneimine (VIII). Yield 7.38 g $(73 \%)$, b.p. - $193-195^{0} \mathrm{C}(1 \mathrm{~mm} \mathrm{Hg})$, $n_{D}^{20}-1.5292, d_{4}^{20}-1.0442 . M R_{D}$ found 99.72 , calculated 100.02. IR spectrum, $v, \mathrm{~cm}^{-1}: 3050$ $\left(\mathrm{C}-\mathrm{N}_{\text {arom. }}\right), 2895\left(\mathrm{CH}_{3}\right), 2830\left(\mathrm{CH}_{2}\right), 1600,1500$ $\left(\mathrm{C}=\mathrm{C}_{\text {arom }}\right), 1200(\mathrm{C}-\mathrm{N}), 1050(\mathrm{C}-\mathrm{O}), 750(\mathrm{C}-\mathrm{S})$. NMR spectrum ${ }^{1} \mathrm{H}, \delta$, ppm: $1.01 \mathrm{t}\left(3 \mathrm{H}, \mathrm{CH}_{3}\right.$, $J=7.1 \mathrm{~Hz}), 1.21-1.32 \mathrm{~m}\left(10 \mathrm{H}, 5 \mathrm{CH}_{2}\right), 2.49 \mathrm{~d} . \mathrm{d}$ $\left(1 \mathrm{H}, \mathrm{CH}_{2} \mathrm{~S}, J=14.0 \mathrm{~Hz}, J=7.7 \mathrm{~Hz}\right), 2.61$ d.d $(1 \mathrm{H}$, $\left.\mathrm{CH}_{2} \mathrm{~S}, J=14.0 \mathrm{~Hz}, J=7.7 \mathrm{~Hz}\right), 3.03 \mathrm{t}(4 \mathrm{H}$, $\left.2 \mathrm{CH}_{2} \mathrm{~N}\right), 3.75 \mathrm{~m}\left(4 \mathrm{H}, 2 \mathrm{CH}_{2} \mathrm{O}\right), 4.04 \mathrm{~m}(1 \mathrm{H}$, $\mathrm{CHO}), 4.51 \mathrm{~s}\left(2 \mathrm{H}, \mathrm{OCH}_{2} \mathrm{~N}\right), 7.15-7.34 \mathrm{~m}(5 \mathrm{H}$, $\left.\mathrm{C}_{6} \mathrm{H}_{5}\right)$. NMR spectrum ${ }^{13} \mathrm{C}, \delta$, ppm: $14.4,15.2$, $17.5,18.2,20.45,21.33,35.4,39.6,69.9,86.4$, 88.15. [M] - 337, calculated - 337.52. Found, $\%$ : C 67.42, $\mathrm{H}$ 9.18, $\mathrm{N}$ 4.10, S 9.41. $\mathrm{C}_{19} \mathrm{H}_{31} \mathrm{NO}_{2} \mathrm{~S}$. Calculated, \%: C 67.61, H 9.26, N 4.15, S 9.50 .

\section{Conclusion}

1. The interaction of phenol with 1-chloropropylthiopropan-2-ol in an alkaline medium at a temperature of $75-80^{\circ} \mathrm{C}$ leads to the formation of 1-phenoxy-3-propylthiopropan-2-ol with a yield of $70 \%$.

2. 1-Phenoxy-3-propylthiopropan-2-ol condenses with formaldehyde and secondary amines at $40-50^{\circ} \mathrm{C}$ for $3-4 \mathrm{~h}$ with the formation of previously unknown aminomethoxy derivatives of 1-phenoxy-3-propylthiopropane in $69-77 \%$ yield.

3. Synthesized aminomethoxy derivatives of 1-phenoxy-3-propylthiopropane possess bactericidal and fungicidal properties and effectively act to suppress the growth of microorganisms in M-11 oil at a concentration of $0.5-1.0 \%$.

4. It has been shown that synthesized aminomethoxy derivatives of 1-phenoxy-3-propylthiopropane exhibit more pronounced antimicrobial activity than drugs used in medical practice alcohol, carbolic acid, rivanol, etc.

The work was carried out on the basis of agreement No 1/18 of April 12, 2018 on scientific and technical cooperation between the Institute of Organo-elemental Compounds named after academician A.N.Nesmeyanov RAS and the Institute of Petrochemical Processes named after academician Yu.G.Mamedaliyev NAS of Azerbaijan.

\section{References}

1. Kuliyev A.M. Khimiya i texnoloqiya prisadok $\mathrm{k}$ maslam i toplivam. M.: Khimiya, 1972. $358 \mathrm{s.}$

2. Nazarov N.M., Sujayev A.R., Garibov E.N. The synthesis of tetrapyrimidines and their Carboxylic Derivatives and the Application of Antimicrobial Properties. J. Chem. and Applied Biochem. 2015. V. 2. No 1. P. 1-4.

3. Mamedbeili G., Dzhafarov I.A., Kochetkov K.A., Kiazimova T.G., Gasanov KH.I., Alieva C.T. Sintez aminometoksiproizvodnyx 1-(benzilsulfanil)pentana // Zh. Org. Khim. 2011. T. 47. № 6. S. 830-832.

4. Golovach N.M., Tkachuk V.N., Sukach V.A., Vovk M.V. Asimmetricheskaya orqanokataliticheskaya reaktsyya Mannikha proizvodnyx 1aril-2,2,2-triftorytilidenkarbaminovoy kisloty $\mathrm{s}$ atsetonom. Zh. Org. Khim. 2012. T. 48. № 9. S. 1188-1191.

5. Kilimochkin Yu.N., Yudashkin A.V., Zhilkina Ye.O., Ivleva Ye.A. Moiseyev I.K., Oshis Ya.F. Odnoreaktornyy metod polucheniya gidroksiproizvodnykh karkasnogo stroyeniya. Zh. Org. Khim. 2017. T. 53. № 7. S. 959-964. 
6. Gulyukina N.S., Makukhin N.N., Beletskaya I.P. Metody sinteza 3(5)-fosfonilirovannykh pirazolov. Uspekhi khimii. 2016. T. 85. № 7. S. 667-683.

7. Kumar S.V., Subramanian M.R., Chinnaiyan S.K. Synthesis, characterisation and evaluation of $\mathrm{N}$ mannich bases of 2-substituted benzimidazole derivatives. J. Young Pharmacists. 2013. V. 5. P. 154-159.

8. Mamedbeyli E.G., Dzhafarov I.A., Ragimova S.K., Gadzhiyeva G.Y. Reaktsiya Mannikha v sinteze biologicheski aktivnykh veshestv. Protsesy neftekhimii i neftepererabotki. 2015. T. 17. № 2 (62). S. 131-158.

9. Subromaniapillali S.G. Mannich reaction: A versatile and convenient approach to bioactive skeletons. J. Chem. Soc. 2013. V. 125. No 3. P. $467-$ 482.

10. Konishi M., Ohkuma H., Tsuno T. et al. Crystal and molecular structure of dynemicin A: a novel 1,5-diyn-3-ene antitumor antibiotic. J. Am. Chem. Soc. 1990. V. 112(9). P. 3715-3716.

11. Grinev A.N., Arkhangelskaya N.V., Uretskaya G.Ya. Izyskaniye farmakologicheski aktivnykh soyedineniy $\mathrm{v}$ ryadu aminometilnykh proizvodnykh 5-oksibenzofutana. Khim. Farm. Zh. 1979. № 3. S. 29-33.

12. Allochio Filho João F., Lemos B.C., De Souza A.S. Greco S.J. Multicomponent Mannich reactions: General aspects, methodologies and applications. Tetrahedron. 2017. V. 73. P. 6977-7004.

13. Mironov V.A., Yankovskiy S.A. Spektroskopiya v orqanicheskoy khimii. Sbornik zadach. M.: Khimiya. 1985. $232 \mathrm{~s}$

14. Yuryev Yu.K. Prakticheskiye raboty po organicheskoy khimii. T. I i II. M.: Izd-vo Moskovskogo un-ta, 1961. $252 \mathrm{~s}$.

\section{1-FENOKSİ-3-PROPILTIOPROPAN-2-OL VӘ İKILİ AMINLOR OSASINDA YENİ MANNIX OSASLARININ SINTEZI}

\section{İ.A.Cəfərov, E.H.Məmmədbəyli, K.A.Koçetkov, A.D.Astanova, G.M.Talıbov}

1-Fenoksi-3-propiltiopropan-2-olun formaldehid və ikili aminlərlə 69-77\% çıxımla onların yeni aminometoksitörəmələrinin alınmasına gətirib çıxaran kondensasiya reaksiyası həyata keçrilmişdir. Alınmış birləşmələrin fizikikimyəvi göstəriciləri təyin edilmiş, quruluşları IQQ, NMR ${ }^{1} \mathrm{H}$ və ${ }^{13} \mathrm{C}$ spektroskopik üsullarla təsdiq edilmişdir. Onların antiseptik xassələri öyrənilmiş və müəyyən edilmişdir ki, hazırda tətbiq olunan preparatlara nisbətən daha yüksək effekt göstərirlər.

Açar sözlar: aminometoksi töramalari, 1-fenoksi-3-propiltiopropan-2-ol, formaldehid, ikili aminlar, dietilamin, dibutilamin, piperidin, morfolin.

\section{СИНТЕЗ НОВЫХ ОСНОВАНИЙ МАННИХА НА ОСНОВЕ 1-ФЕНОКСИ-3-ПРОПИЛТИОПРОПАН-2- ОЛА И ВТОРИЧНЫХ АМИНОВ}

\section{И.А.Джафаров, Э.Г.Мамедбейли, К.А.Кочетков, А.Д.Астанова, Г.М.Талыбов}

Осуществлена конденсация 1-фенокси-3-пропилтиопропан-2-ола с формальдегидом и вторичными алифатическими, а также гетероциклическими аминами, которая привела к образованию новых аминометоксипроизводных 1-фенокси-3-пропилтиопропана с 69-77\%-ными выходами. Определены физико-химические показатели синтезированных соединений, их строение подтверждено методами элементного анализа, ИК-спектроскопии, ЯМР ${ }^{1} \mathrm{H}$ и ${ }^{13} \mathrm{C}$. Полученные соединения испытаны в качестве антисептических веществ против бактерий и грибов. Установлено, что они являются более эффективными, чем применяемые в настоящее время медицинские препараты.

Ключевые слова: аминометоксипроизводные, 1-фенокси-3-пропилтиопропан-2-ол, формальдегид, вторичные амины, диэтиламин, дибутиламин, пиперидин, морфолин. 